\title{
Thoracic Radiological Characteristics of COVID-19 Patients at the Time of Presentation: A Cross-sectional Study
}

\author{
Srikant Behera ${ }^{1}$, Souvik Maitra ${ }^{2}$, Rahul K Anand ${ }^{3}$, Dalim K Baidya ${ }^{4}$, Rajeshwari Subramaniam ${ }^{5}$, Choro A Kayina ${ }^{6}$, \\ Bikash R Ray ${ }^{7}$
}

\begin{abstract}
Background: Coronavirus disease 2019 (COVID-19) is a type of pneumonia caused by severe acute respiratory syndrome coronavirus 2 (SARSCoV-2). COVID-19 pneumonia has characteristic radiological features. Recent evidence indicates usefulness of chest X-ray and lung ultrasound (LUS) in detecting COVID-19 pneumonia.

Materials and methods: In this prospective observational study, chest X-ray and LUS features of 50 adults with COVID-19 pneumonia at the time of presentation were described.

Results: Chest X-ray findings were present in $96 \%$ of patients, whereas all patients have ultrasound finding. Proportion (95\% Cl) of patients having bilateral opacities in chest X-ray was $96 \%$ (86.5-98.9\%), ground glass opacity $74 \%$ (60.5-84.1\%), and consolidation 50\% (36.7-63.4\%). In LUS, shred sign and thickened pleura was present in all patients recruited in this study. Air bronchogram was present in at least one area in $80 \%$ of all patients and B-lines score of more than 2 was present in at least one lung area in $84 \%$ patients. Number of lung areas with "shred sign" were higher in hypoxemic $(p=0.005)$ and tachypneic $(p=0.006)$ patients and pleura line abnormalities were present in more lung areas in hypoxemic patients $(p=0.03)$.

Conclusion: According to our study, LUS is a useful tool not only in diagnosing, but it also correlates with requirement of respiratory support in COVID-19 patients.
\end{abstract}

Keywords: Chest X-ray, COVID-19, Lung ultrasound, Severe acute respiratory syndrome coronavirus 2, Shred sign.

Indian Journal of Critical Care Medicine (2021): 10.5005/jp-journals-10071-23705

\section{INTRODUCTION}

In December 2019, a series of pneumonia cases of unknown cause emerged in Wuhan, Hubei, China, which was later found to be a novel coronavirus. ${ }^{1}$ Early reports from Wuhan described the associated coronavirus disease 2019 (COVID-19) as an atypical pneumonia in which $26-33 \%$ of patients required intensive care and 4-15\% mortality. ${ }^{2}$ COVID-19 pneumonia has characteristic features that can be studied radiologically. Computer tomography (CT) scan finding has high sensitivity but lacks specificity for COVID-19 and hence has a good screening potential. ${ }^{3}$ Signs on chest X-ray and lung ultrasound (LUS) are nonspecific when considered alone; however, combinations of patterns and distribution of signs in different lung field may allow a reliable characterization of the disease. ${ }^{4-6}$ In this study we investigate the chest X-ray and lung US characteristics of COVID-19 patients at the time of presentation to the hospital and try to find out any association between US characteristics with requirement of respiratory support.

\section{Materials and Methods}

This prospective observational study was conducted at the COVID area and ICU of All India Institute of Medical Sciences, New Delhi, after institute ethical committee clearance. Real-time RT-PCR of 50 adults (aged between 18 and 80 years) confirmed COVID-19 patients were included in the study. Patients who refused to give consent were excluded. Data related to baseline demographics, comorbidities, symptoms, presence of hypoxia (oxygen saturation $<92 \%$ ), and requirement of organ support (respiratory, hemodynamic, renal) were collected at the time of admission. After confirmation of COVID-19 infection, chest X-ray

\footnotetext{
${ }^{1-7}$ Department of Anaesthesiology, Pain Medicine and Critical Care, All India Institute of Medical Sciences, New Delhi, India
}

Corresponding Author: Bikash R Ray, Department of Anaesthesiology, Pain Medicine and Critical Care, All India Institute of Medical Sciences, New Delhi, India, Phone: +919899323110, e-mail: bikashray.aiims@ gmail.com

How to cite this article: Behera S, Maitra S, Anand RK, Baidya DK, Subramaniam R, Kayina CA, et al. Thoracic Radiological Characteristics of COVID-19 Patients at the Time of Presentation: A Cross-sectional Study. Indian J Crit Care Med 2021;25(1):85-87.

Source of support: Nil

Conflict of interest: None

and point-of-care lung US scanning [12 zones ( 6 in each lung)] was done in all the patients. ${ }^{7}$ The ultrasound parameters were measured using an ultrasound device (Sonosite M-Turbo Ultrasound System, SonoSite, Inc. Bothell, WA, USA).

Chest X-ray was reported in terms of ground glass opacity and consolidations in upper, middle, and lower zones. Each of these zones occupies approximately one-third of the height of the lungs. All lung zones were scanned for B-lines, pleural line abnormalities (irregular and/or thickened pleura), air bronchogram, subpleural consolidation, and pleural effusion. B-line score was recorded as per number of $B$-lines $(0=<3$ per image, $1=3-7$ per rib space, $2=>7$ per rib space, $3=$ confluent $B$-lines). ${ }^{8}$ Interpretation of chest $X$-ray and lung US scanning were performed by four experienced intensivists involved in the study. Severity of the disease (WHO

(c) The Author(s). 2021 Open Access This article is distributed under the terms of the Creative Commons Attribution 4.0 International License (https://creativecommons. org/licenses/by-nc/4.0/), which permits unrestricted use, distribution, and non-commercial reproduction in any medium, provided you give appropriate credit to the original author(s) and the source, provide a link to the Creative Commons license, and indicate if changes were made. The Creative Commons Public Domain Dedication waiver (http://creativecommons.org/publicdomain/zero/1.0/) applies to the data made available in this article, unless otherwise stated. 
classification), oxygen requirement, and type of respiratory support $\left(\mathrm{O}_{2}\right.$ by face mask, NIV, HFNC, mechanical ventilation, etc.) were recorded at the point of enrolment to the study. All analyses were performed using STATA version 13 for Mac OS (StataCorp. 2011. Stata Statistical Software: Release 13. College Station, TX: StataCorp LP).

\section{Results}

In this observational study, fifty COVID-19-positive patients' data were analyzed who have a median interquartile range (IQR) age of $51.5(42-64)$ years and $78 \%$ of them were male. At the time of presentation, $86 \%$ patients were febrile, $84 \%$ had shortness of breath, and $68 \%$ cough. Median (IQR) duration of symptom was $4^{3-6}$ days. Chest pain, myalgia, and fatigue were infrequent and present in 10,4, and $14 \%$ patients, respectively. Hypertension was the commonest associated comorbidity and was present in $32 \%$ patients followed by diabetes (22\%), chronic kidney disease (16\%), and coronary artery disease (10\%).

Proportion $(95 \% \mathrm{Cl})$ of patients having bilateral opacities in chest X-ray was $96 \%$ (86.5-98.9\%), ground glass opacity $74 \%$ (60.5-84.1\%), and consolidation 50\% (36.7-63.4\%). Only 6\% patients had pleural effusion evident in chest $X$-ray. Presence of ground glass opacity was similar in hypoxemic and nonhypoxemic patients ( $p$ $=0.75)$ and also in different respiratory support groups $(p=0.08$, Fisher exact test). Details of chest $X$-ray involvement were reported in Table 1.

In lung US, shred sign and thickened pleura were present in all patients recruited in this study. Air bronchogram was present in at least one area in $80 \%$ of all patients and B-line score of $>2$ was present at least one lung area in $84 \%$ patients. Pleural effusion was detected in $8 \%$ patients with lung US. Details of lung US findings are provided in Table 2. Number of lung areas with "shred sign"

Table 1: Pattern of involvement in chest X-ray [data represented as proportion $(95 \% \mathrm{Cl})]$

\begin{tabular}{lll}
\hline & Right & Left \\
\hline Upper zone & $58(44.2-70.6)$ & $58(44.2-70.6)$ \\
Middle zone & $88(76.2-94.4)$ & $78(64.8-87.3)$ \\
Lower zone & $88(76.2-94.4)$ & $86(73.8-93.1)$ \\
\hline
\end{tabular}

was higher in hypoxemic $(p=0.005)$ and tachypnoeic $(p=0.006)$ patients and pleura line abnormalities were present in more lung areas in hypoxemic patients $(p=0.03)$. Total number of B-lines was higher in patients with tachypnea $(p=0.04)$. Total number of areas with "shred sign" were higher with increasing degree of respiratory support ( $p=0.003$ ). Distribution of US features in different groups of patients has been described in Table 3 and Figure 1. Ordinal logistic regression revealed that presence of thickened pleura was associated with requirement organ support [odds ratio $(95 \% \mathrm{Cl})$ $1.7(1.19-2.62)]$.

\section{Discussion}

Among 50 patients with moderate to severe/critical COVID-19, chest X-ray findings were present in $96 \%$ of patients, whereas $100 \%$ of the patients have US finding suggestive of COVID-19. Chest X-ray finding in our study shows bilateral involvement, with middle and lower zones being more commonly affected. Similar observations were made in lung ultrasound where bilaterally zones 4 and 6 have more consistent finding suggestive of COVID-19. These findings are in accordance with previous published chest X-ray and CT scan finding. ${ }^{3,4}$ Involvement of posterior basal zones explains the efficacy of prone positioning in improving oxygenation in many of these patients.

Higher number of areas with subpleural shred sign and pleural line abnormalities were found to be correlating with the severity of the disease and requirement of respiratory support. In critically ill patients, Xing et al. also have documented increased B-lines and consolidations. ${ }^{9}$ Patients with higher areas of shredding and high total B-line scores were more tachypnoeic. This highlights the importance of early lung US, which can help in deciding the type of respiratory support. Pleural effusion was diagnosed in $8 \%$ of cases by US, and all these patients had mild pleural effusion. Large pleural effusions with COVID-19 are rare and if present suggests associated bacterial infection or heart failure.

Radiological characteristics of all the patients at initial presentation were evaluated. However, there are certain limitation of the study. First, the progression of finding on X-ray and lung US over the course of hospitalization was not assessed. Second, X-ray and lung US findings were not independently assessed by blinded radiologist and interpreted by clinicians involved in patient care.

Table 2: Pattern of lung ultrasound characteristics [data presented as median (IQR) or proportion (95\% Cl) as applicable]

\begin{tabular}{rllcc}
\hline & \multicolumn{3}{c}{ USG findings } \\
\cline { 2 - 5 } & B-lines score & Shred sign & Pleural line abnormalities & Air bronchogram \\
\hline Right & & & & \\
Zone 1 & $0(0-0)$ & $50(36.7-63.4)$ & $44(31.2-57.7)$ & $0(0-7.1)$ \\
Zone 2 & $0(0-1)$ & $52(38.5-65.2)$ & $56(42.3-68.8)$ & $6(2.1-16.2)$ \\
Zone 3 & $0.5(0-1)$ & $62(48.2-74.1)$ & $20(11.2-33)$ & $12(5.6-23.8)$ \\
Zone 4 & $1(1-2)$ & $78(64.8-87.3)$ & $70(56.3-80.9)$ & $36(24.1-49.9)$ \\
Zone 5 & $1(0-2)$ & $46(32.9-59.6)$ & $16(8.3-28.5)$ & $20(11.2-33)$ \\
Zone 6 & $2(1-2)$ & $48(34.8-61.5)$ & $42(29.4-55.8)$ & $40(27.6-53.8)$ \\
Left & & & & \\
Zone 1 & $0(0-1)$ & $54(40.4-67)$ & $38(25.9-51.9)$ & $0(0-7.1)$ \\
Zone 2 & $1(0-1)$ & $48(34.8-61.5)$ & $66(52.2-77.6)$ & $4(1.1-13.5)$ \\
Zone 3 & $1(0-1)$ & $64(50.1-75.9)$ & $14(6.9-26.2)$ & $10(4.4-21.4)$ \\
Zone 4 & $2(1-2)$ & $68(54.2-79.2)$ & $52(38.5-65.2)$ & $46(33-59.6)$ \\
Zone 5 & $1(1-2)$ & $46(33-59.6)$ & $8(3.2-18.8)$ & $14(6.9-26.2)$ \\
Zone 6 & $2(2-3)$ & $42(29.4-55.8)$ & $26(15.9-39.6)$ & $58(44.2-70.6)$ \\
\hline
\end{tabular}


Table 3: Pattern of lung ultrasound across different levels of respiratory support

\begin{tabular}{|c|c|c|c|c|c|}
\hline & $\begin{array}{l}\text { Without supplementary } \\
\text { oxygen }(n=3)\end{array}$ & $\begin{array}{l}\text { Oxygen by facemask } \\
(n=4)\end{array}$ & $\operatorname{HFNC/NIV~}(n=15)$ & $\begin{array}{l}\text { Mechanically } \\
\text { ventilated }(n=28)\end{array}$ & pvalue \\
\hline Total B-lines score & $11(3-23)$ & $15(10-18.5)$ & $11(6-17)$ & $13(10-17.5)$ & 0.54 \\
\hline Shred sign $\$$ & $2(2-4)$ & $5.5(4.5-6)$ & $7(5-9)$ & $6.5(5.5-8)$ & 0.003 \\
\hline Pleural line abnormalities (PLA) & $2(2-3)$ & $5(4-6.5)$ & $4(3-4)$ & $5(4-6)$ & 0.88 \\
\hline Air bronchogram $\$$ & $0(0-4)$ & $2(0-4.5)$ & $3(1-5)$ & $2(1-3.5)$ & 0.23 \\
\hline
\end{tabular}

\$Number of lung areas involved

${ }^{\#}$ Nonparametric test for trend across ordered groups

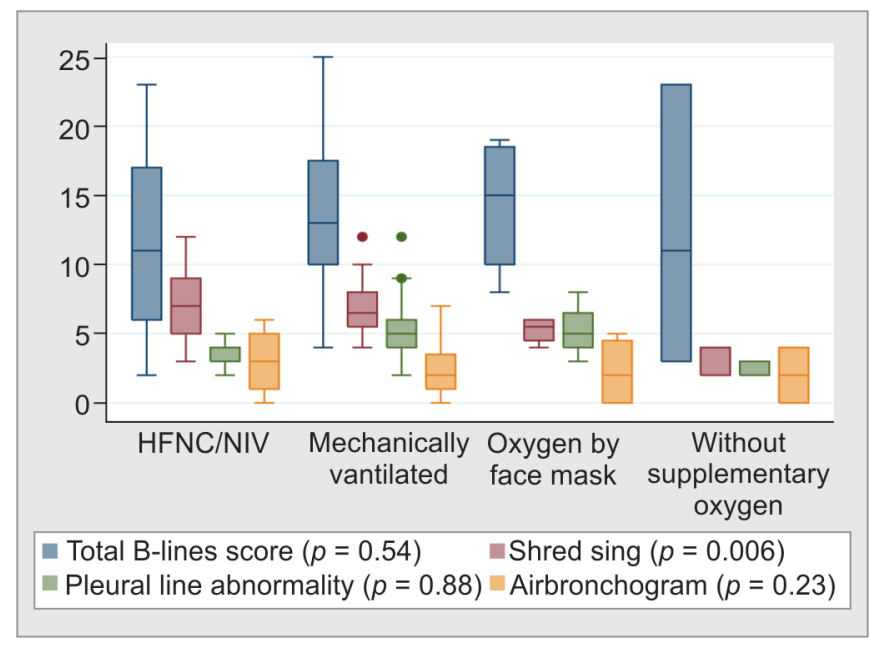

Fig. 1: Box-Whisker plot showing median (IQR) of total B-line score, number of areas with shred sign, pleural line abnormalities, and air bronchogram in patients with different degree of respiratory support

Last, data related to final outcome of these patients were not collected.

\section{Conclusion}

In summary, lung US signs of COVID-19 are present in all patients at admission to the hospital. The pattern of involvement is specific, mainly manifested by pleural line abnormalities, interstitial lesions, subpleural consolidations, and primarily involve peripheral posterior basal zones, bilaterally. Number of areas with subpleural "shred sign" correlates with severity of disease. According to our study, LUS is a useful tool not only in diagnosing, but it also correlates with requirement of respiratory support in COVID-19 patients.

\section{References}

1. Huang C, Wang Y, Li X, Ren L, Zhao J, Hu Y, et al. Clinical features of patients infected with 2019 novel coronavirus in Wuhan, China. Lancet 2020;395(10223):497-506. DOI: 10.1016/S0140-6736(20)301835.

2. Zhu N, Zhang D, Wang W, Li X, Yang B, Song J, et al. A novel coronavirus from patients with pneumonia in China, 2019. N Engl J Med 2020;382(8):727-733. DOI: 10.1056/NEJMoa2001017.

3. Fang $Y$, Zhang $H$, Xie J, Lin M, Ying L, Pang P, et al. Sensitivity of chest CT for COVID-19: comparison to RT-PCR. Radiology 2020. 200432. (In press).

4. Cao Y, Liu X, Xiong L, Cai K. Imaging and clinical features of patients with 2019 novel coronavirus SARS-CoV-2: a systematic review and meta-analysis. J Med Virol 2020. 10. (In Press).

5. Wong HYF, Lam HYS, Fong AH, Leung ST, Chin TWY, Lo CSY, et al. Frequency and distribution of chest radiographic findings in COVID19 positive patients. Radiology 2019. 201160. (In Press).

6. Peng QY, Wang XT, Zhang LN, Chinese Critical Care Ultrasound Study Group (CCUSG) Findings of lung ultrasonography of novel corona virus pneumonia during the 2019-2020 epidemic. Intensive Care Med 2020;46(5):849-850. DOI: 10.1007/s00134-020-05996-6.

7. Mojoli F, Bouhemad B, Mongodi S, Lichtenstein D. Lung ultrasound for critically ill patients. Am J Respir Crit Care Med 2019;199(6):701-714. DOI: $10.1164 / \mathrm{rccm} .201802-0236 \mathrm{Cl}$.

8. Brusasco C, Santori G, Bruzzo E, Tro R, Robba C, Tavazzi G, et al. Quantitative lung ultrasonography: a putative new algorithm for automatic detection and quantification of B-lines. Crit Care 2019;23(1):288. DOI: 10.1186/s13054-019-2569-4.

9. Xing C, Li Q, Du H, Kang W, Lian J, Yuan L. Lung ultrasound findings in patients with COVID-19 pneumonia. Crit Care 2020;24(1):174. DOI: 10.1186/s13054-020-02876-9. 